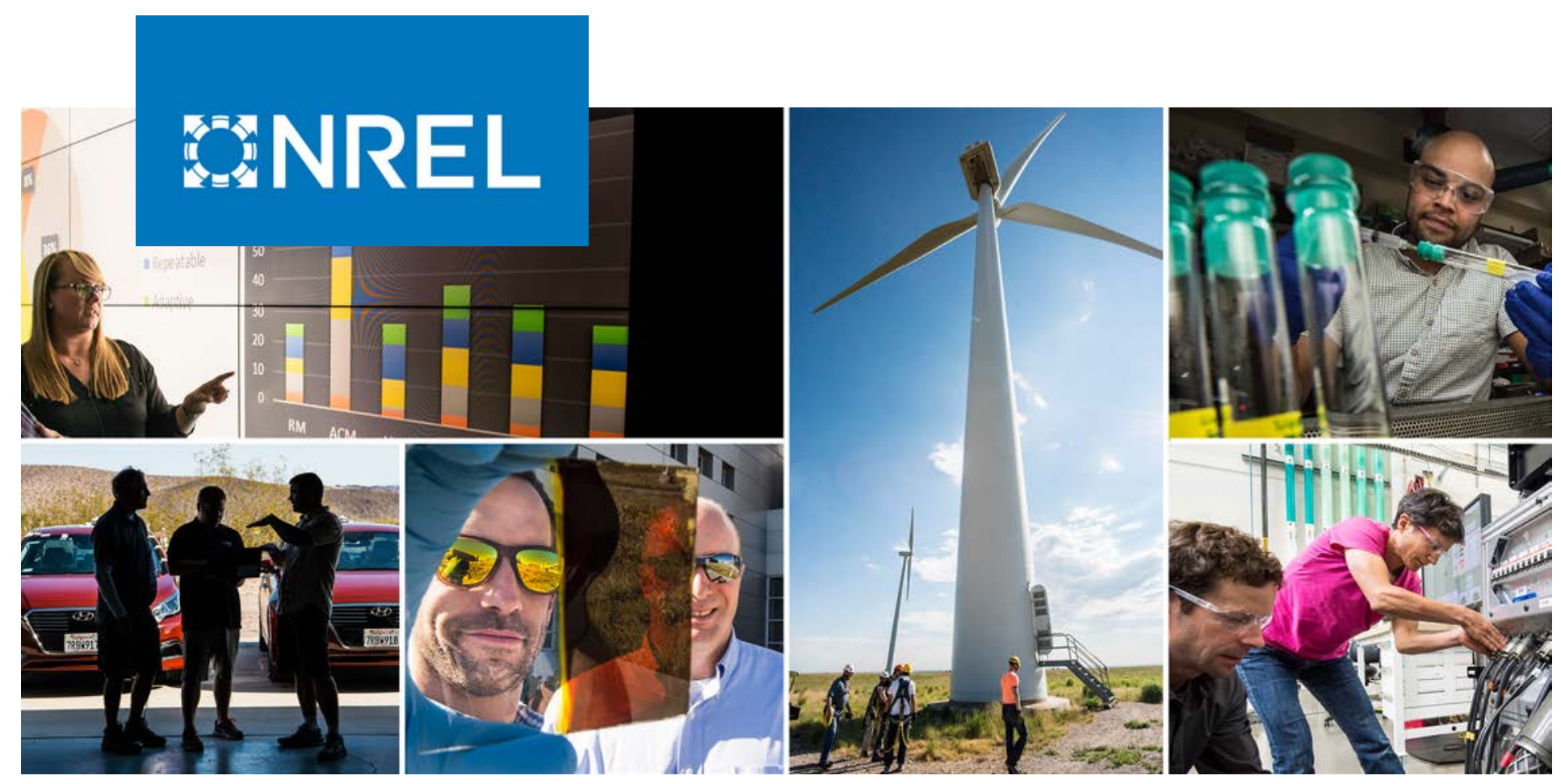

\title{
AC Resistance Reduction Using Orthogonal Air Gaps in High-Frequency Inductors
}

\section{Preprint}

Satyaki Mukherjee, ${ }^{1}$ Yucheng Gao, ${ }^{1}$ Regina Ramos, ${ }^{2}$ Vivek Sankaranarayanan, ${ }^{1}$ Branko Majmunovic, ${ }^{1}$ Rahul Mallik, ${ }^{3}$ Soham Dutta, ${ }^{3}$ Gab-Su Seo, ${ }^{4}$ Brian Johnson, ${ }^{3}$ and Dragan Maksimovic ${ }^{1}$

${ }^{1}$ University of Colorado, Boulder

${ }^{2}$ Polytechnic University of Madrid

${ }^{3}$ University of Washington

${ }^{4}$ National Renewable Energy Laboratory

Presented at the 2019 IEEE Workshop on Control and Modeling for Power Electronics (IEEE COMPEL)

Toronto, Canada

June 17-20, 2019

NREL is a national laboratory of the U.S. Department of Energy

Office of Energy Efficiency \& Renewable Energy

Operated by the Alliance for Sustainable Energy, LLC

This report is available at no cost from the National Renewable Energy Laboratory (NREL) at www.nrel.gov/publications.
Conference Paper

NREL/CP-5D00-74060

September 2019 


\title{
GNREL
}

\section{AC Resistance Reduction Using Orthogonal Air Gaps in High-Frequency Inductors}

\section{Preprint}

\author{
Satyaki Mukherjee, ${ }^{1}$ Yucheng Gao, ${ }^{1}$ Regina Ramos, ${ }^{2}$ \\ Vivek Sankaranarayanan, ${ }^{1}$ Branko Majmunovic, ${ }^{1}$ \\ Rahul Mallik, ${ }^{3}$ Soham Dutta, ${ }^{3}$ Gab-Su Seo, ${ }^{4}$ \\ Brian Johnson, ${ }^{3}$ and Dragan Maksimovic ${ }^{1}$ \\ ${ }^{1}$ Colorado Power Electronics Center \\ 2 Polytechnic University of Madrid \\ ${ }^{3}$ University of Washington \\ ${ }^{4}$ National Renewable Energy Laboratory
}

\section{Suggested Citation}

Mukherjee, Satyaki, Yucheng Gao, Regina Ramos, Vivek Sankaranarayanan, Branko Majmunovic, Rahul Mallik, Soham Dutta, Gab-Su Seo, Brian Johnson, and Dragan Maksimovic. 2019. AC Resistance Reduction Using Orthogonal Air Gaps in HighFrequency Inductors: Preprint. Golden, CO: National Renewable Energy Laboratory. NREL/CP-5D00-74060. https://www.nrel.gov/docs/fy19osti/74060.pdf.

(C) 2019 IEEE. Personal use of this material is permitted. Permission from IEEE must be obtained for all other uses, in any current or future media, including reprinting/republishing this material for advertising or promotional purposes, creating new collective works, for resale or redistribution to servers or lists, or reuse of any copyrighted component of this work in other works.

NREL is a national laboratory of the U.S. Department of Energy Office of Energy Efficiency \& Renewable Energy Operated by the Alliance for Sustainable Energy, LLC

This report is available at no cost from the National Renewable Energy Laboratory (NREL) at www.nrel.gov/publications.

Contract No. DE-AC36-08GO28308
Conference Paper NREL/CP-5D00-74060 September 2019

National Renewable Energy Laboratory 15013 Denver West Parkway Golden, CO 80401

303-275-3000 • www.nrel.gov 


\section{NOTICE}

This work was authored in part by the National Renewable Energy Laboratory, operated by Alliance for Sustainable Energy, LLC, for the U.S. Department of Energy (DOE) under Contract No. DE-AC36-08GO28308. Funding provided by U.S. Department of Energy Office of Energy Efficiency and Renewable Energy Solar Energy Technologies Office and the Laboratory Directed Research and Development (LDRD) Program. The views expressed herein do not necessarily represent the views of the DOE or the U.S. Government. The U.S. Government retains and the publisher, by accepting the article for publication, acknowledges that the U.S. Government retains a nonexclusive, paid-up, irrevocable, worldwide license to publish or reproduce the published form of this work, or allow others to do so, for U.S. Government purposes.

This report is available at no cost from the National Renewable Energy Laboratory (NREL) at www.nrel.gov/publications.

U.S. Department of Energy (DOE) reports produced after 1991 and a growing number of pre-1991 documents are available free via www.OSTI.gov.

Cover Photos by Dennis Schroeder: (clockwise, left to right) NREL 51934, NREL 45897, NREL 42160, NREL 45891, NREL 48097, NREL 46526.

NREL prints on paper that contains recycled content. 


\title{
AC Resistance Reduction Using Orthogonal Air Gaps in High Frequency Inductors
}

\author{
Satyaki Mukherjee*, Yucheng Gao*, Regina Ramos ${ }^{\dagger}$, Vivek Sankaranarayanan*, Branko Majmunovic* \\ Rahul Mallik ${ }^{\ddagger}$, Soham Dutta ${ }^{\ddagger}$, Gab-Su Seo ${ }^{\S}$, Brian Johnson ${ }^{\ddagger}$, and Dragan Maksimović* \\ * Colorado Power Electronics Center \\ Department of Electrical, Computer and Energy Engineering \\ University of Colorado, Boulder, CO 80309-425, USA \\ Email: \{satyaki.mukherjee, yucheng.gao, vivek.sankaranarayanan, branko.majmunovic, maksimov\}@ colorado.edu \\ ${ }^{\dagger}$ Centro de Electrónica Industrial (CEI), Universidad Politécnica de Madrid (UPM) \\ Email: regina.ramos@upm.es \\ $\ddagger$ University of Washington \\ Email: \{rmallik, sdutta, brianbj\}@uw.edu \\ ${ }_{\S}$ Power Systems Engineering Center, National Renewable Energy Laboratory, Golden, CO 80401, USA \\ Email: gabsu.seo@nrel.gov
}

\begin{abstract}
This paper presents a relatively simple technique to reduce winding losses due to fringing fields in high-frequency inductors. In high-frequency power electronics, ac inductor winding losses are affected by skin and proximity effects, including uneven current distribution due to fringing magnetic fields around airgaps. It is well known how fringing effects can be mitigated using distributed airgaps, at the expense of non-standard core or winding geometry. In planar magnetics, the orthogonal-airgap approach proposed in this paper combines airgaps in core segments parallel with the windings with conventionally placed airgaps in segments perpendicular to the windings. The approach is developed using a 1D analytical framework and validated by 2D finite-element simulations. Analytical guidelines are presented to optimize the airgaps to achieve minimum ac resistance. As a case study, a planar inductor is designed for an $8 \mathrm{~kW} \mathrm{SiC}$ based buck converter operating at $250 \mathrm{kHz}$. It is shown how the orthogonal airgaps result in more than $45 \%$ reduction in ac resistance and substantially reduced inductor losses compared to the design using standard airgaps. The results are verified by loss measurements on an experimental converter prototype.
\end{abstract}

\section{INTRODUCTION}

Ac losses, including core losses as well as winding losses due to skin and proximity effects, limit the performance of inductors in high-frequency power electronics [1]-[4]. In addition, magnetic structures with airgaps lead to higher ac resistance and higher ac winding losses because of uneven current distribution due to fringing magnetic fields around

Funding for the work reported in this paper was provided in part by the U.S Department of Energy (DOE), Office of E nergy E fficiency an d Renewable Energy, Solar Energy Technologies Office grant number DE-EE0008346. This work was authored in part by the National Renewable Energy Laboratory, operated by Alliance for Sustainable Energy, LLC, for the U.S. DOE under Contract No. DE-AC36-08GO28308. The views expressed in the article do not necessarily represent the views of the DOE or the U.S. Government.

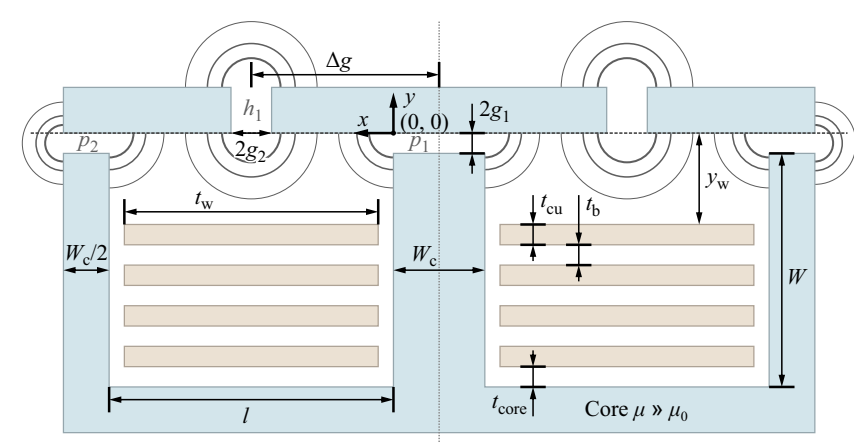

Figure 1: Planar inductor structure with orthogonal airgaps, including standard perpendicular airgaps $p$, and parallel airgaps $h$.

the airgaps [5]-[7]. Many prior works have been focused on mitigating the fringing effects by distributing the airgaps along the magnetic path, predominantly in segments of the core parallel with the windings [5]-[9]. In general, these approaches rely on numerical finite-element based design optimization and often require nonstandard core or winding arrangements, which may complicate manufacturing.

This paper describes a relatively simple approach based on orthogonally placed airgaps, comprising standard airgaps in core segments perpendicular to the windings, and airgaps in core segments parallel with the windings, as illustrated by the planar inductor structure of Fig. 1. A scalar potential-based 1D analytical approach described in [10] is used to develop an intuitive analytical understanding of how the orthogonal airgaps result in more uniform current distribution and substantial reduction in ac resistance. Furthermore, the analytical approach allows for relatively simple design optimization.

The paper is organized as follows: Section II derives the scalar potential based expressions for fringing fields in the geometry shown in Fig. 1. A planar inductor case study, 


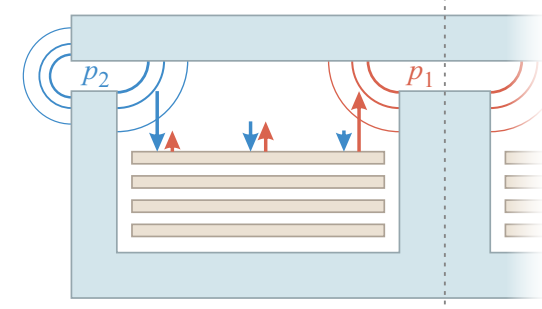

(a) Perpendicular airgaps

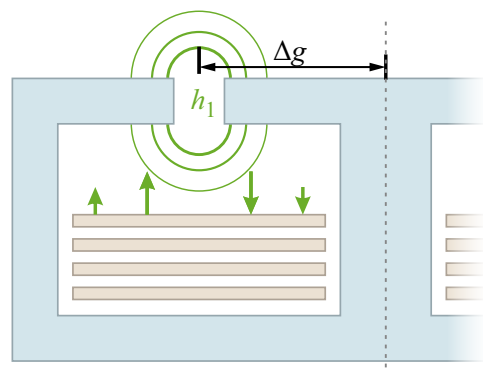

(b) Parallel airgap

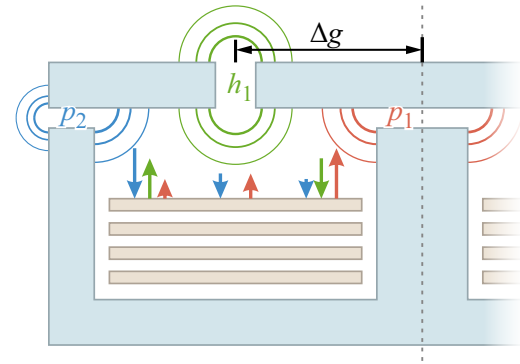

(c) Orthogonal airgaps $\begin{aligned} & \text { H fields } \\ - & H_{p 1, y}(x, y) \\ - & H_{h 1, y}(x, y) \\ - & H_{p 2, y}(x, y)\end{aligned}$

(

Figure 2: Effective fringing $H$ fields in a planar EI inductor structure with (a) conventional (perpendicular) airgaps $p$, (b) parallel airgaps $h$, and (c) orthogonal airgaps $p$ and $h$.

including analytical results and 2D simulation validations, is provided in Section III. Additional effects of $H$-fields due to winding currents, and optimization of gap lengths are also addressed in this section. Experimental results presented in Section IV confirm more than $45 \%$ reduction in ac winding losses compared to the design with standard airgaps. Section V concludes the paper.

\section{Analytical Framework}

Figure 2 shows three planar inductor structures using EI core segments: (a) a conventional structure with standard perpendicular airgaps $p_{1}, p_{2}$, (b) a structure with parallel (horizontal) airgaps $h_{1}$ [2], [5] and (c) a structure with orthogonal airgaps. The three cases are compared in terms of the $\mathrm{H}$ field distribution, current distribution, and ac resistance. It is assumed that identical planar windings and airgaps are selected to obtain the same inductance.

Current density in the top winding layer depends only on the $H$-field component perpendicular to the conductor, denoted as $H_{p 1, y}, H_{p 2, y}$ and $H_{h 1, y}$ in Fig. 2, which illustrates the intuition for the advantages and disadvantages of the alternative gap arrangements. As shown in Fig. 2(c), the effective fringing fields originated from the perpendicular gaps $p_{1}, p_{2}$ and the parallel gap $h_{1}$ can be decomposed in the $x$ and $y$ directions following the scalar potential-based 1D method developed in [10]. One may note that $H_{h 1, y}$ (y component of the fringing field produced by gap $h_{1}$ ) acts against the dominant $H$ field component at both conductor edges, which reduces the effect of current crowding. This field cancellation is in contrast to the case of standard airgaps shown in Fig. 2(a), where the large components of the $H$ field result in current crowding at the conductor edges.

Moving the gap to the core segment parallel to the windings, as shown in Fig. 2(b) helps mitigate the problem of current crowding at the edges, as discussed in [5]. Compared to Fig. 2(b), the orthogonal airgap approach of Fig. 2(c) offers further improvements in two ways: first, the airgaps are shorter, which reduces magnitude of the fringing fields, and second, the field cancellation further improves uniformity. At the expense of increased complexity, the approach of Fig. 2(b) can be further improved by distributing additional airgaps along the parallel segments of the core [5], [11]. In all cases, however, the orthogonal airgap approach, which amounts to simple addition of conventionally placed airgaps using a spacer between core segments, offers further improvements without the need to increase the number of core segments or complexity of the assembly.

Referring to Fig. 1, key geometrical parameters are the gap lengths $2 g_{1}$ and $2 g_{2}$, position $\Delta g$, distance $y_{w}$ from the top surface of the top winding layer to the parallel core segment, distance $t_{\text {core }}$ of the windings from the core, winding thickness $t_{c u}$, spacing $t_{b}$ between the turns, and turn width $t_{w}=l-$ $2 t_{\text {core }}$. The core parameters are $l, W_{c}$ and $W$.

The field components of interest at the face of the top winding layer ( $y_{w}$ below the I segment of the core) are [10]:

$$
\begin{aligned}
& H_{g 1}=H_{g 2}=\frac{0.9 N I}{2\left(2 g_{1}+g_{2}\right)} \\
& H_{p 1, y}=-\frac{H_{g 1}}{\pi} \tan ^{-1}\left(\frac{4 x g_{1}}{x^{2}+y_{w}^{2}-4 g_{1}^{2}}\right) \\
& H_{p 2, y}=-\frac{H_{g 1}}{\pi} \tan ^{-1}\left(\frac{4(l-x) g_{1}}{(l-x)^{2}+y_{w}^{2}-4 g_{1}^{2}}\right) \\
& H_{h 1, y}=\frac{H_{g 2}}{2 \pi} \ln \left[\frac{y_{w}^{2}+\left(x-\Delta g+g_{2}\right)^{2}}{y_{w}^{2}+\left(x-\Delta g-g_{2}\right)^{2}}\right]
\end{aligned}
$$

where

$$
t_{\text {core }} \leq x \leq l-t_{\text {core }}
$$

From the analytical expressions in (1), the two-fold advantages of the orthogonal airgaps can be analyzed. First, to get the same inductance, the additional gaps are shorter, which results in reduced fringing fields, as is the case in all distributed-gap techniques. More importantly, since $H_{h 1, y}$ opposes $H_{p 1, y}$ and $H_{p 2, y}$ at the two conductor edges, respectively, a more uniform distribution of the $H$ field is obtained, resulting in more uniform current distribution and thereby reduced ac resistance. To illustrate this point further, the $y$-direction fringing $H$-fields due to the airgap in the three different arrangements shown in Fig. 2 are plotted in Fig. 3. The resultant fringing field is most uniform across the face of the conductor in the orthogonal airgap case of Fig. 2(c), which results in the lowest inductor ac resistance.

\section{CASe Study: High-Frequency Planar Inductor}

To illustrate and validate the orthogonal gap approach of Section II, consider an $8 \mu \mathrm{H}$ planar inductor built using 


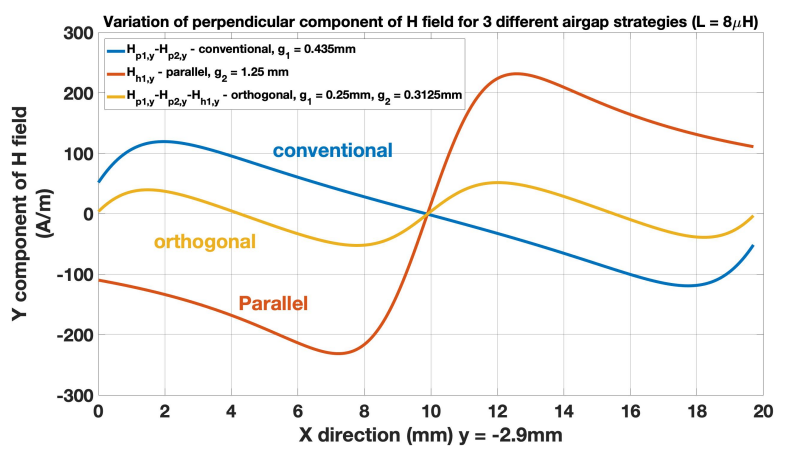

Figure 3: Distribution of the fringing $H$-field component perpendicular to the winding for the three airgap arrangements shown in Fig. 2.

EILP 64 core set including ELP 64/10/50 E-shaped segment and I 64/5/50 I-shaped segment, with 4 turns on a 4-layer PCB having $4 \mathrm{oz}$ copper thickness. The inductor parameters are as follows:

$$
\begin{aligned}
& N=4 ; I=1 ; t_{c u}=0.14 \mathrm{~mm} ; \\
& t_{\text {core }}=1 \mathrm{~mm} ; t_{b}=0.25 \mathrm{~mm} ; \\
& W=5.1 \mathrm{~mm} ; W_{c}=10.2 \mathrm{~mm} ; l=21.7 \mathrm{~mm} ;
\end{aligned}
$$

Three inductor designs are considered based on the three airgap arrangements shown in Fig. 2. The corresponding magnetic circuit models are shown in Fig. 4. In all cases, the gaps are such that the inductance takes on the same value of $8, \mu \mathrm{H}$, which uniquely determines the gap lengths $g_{1}$ and $g_{2}$ in Fig. 4(a) and Fig. 4(b), respectively. In the orthogonal airgap case, selection of the gap lengths, $g_{1}$ and $g_{2}$, and the position, $\Delta g$, can be considered a constrained optimization problem, which is addressed in the subsequent sections.

\section{A. H-fields due to winding currents}

The $H$-field contributions due to fringing are described by (1). To complete the analytical model, it is necessary to consider the $H$-field effects due to the winding currents. One

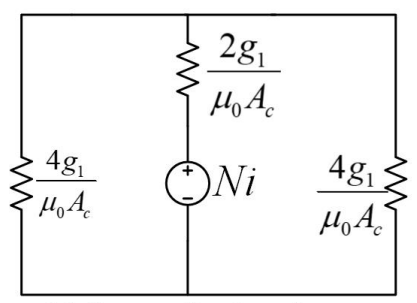

(a) Perpendicular airgaps

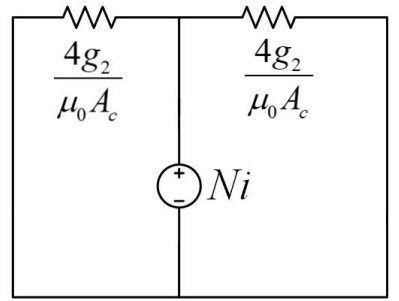

(b) Parallel airgaps

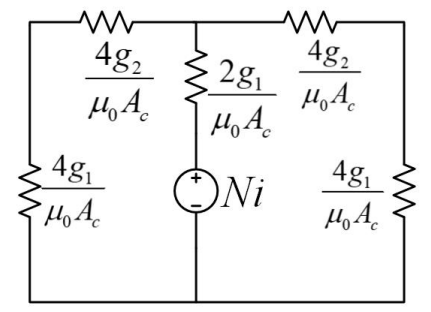

(c) Orthogonal airgaps

Figure 4: Equivalent magnetic circuit models for the considered airgap arrangements.

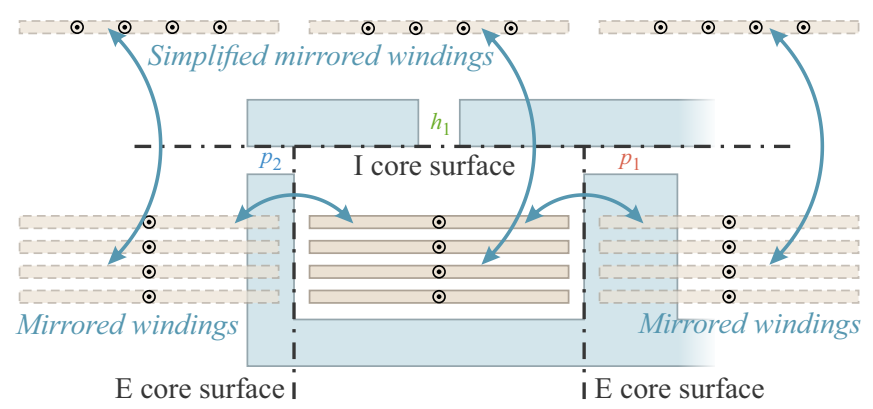

Figure 5: Equivalent winding arrangement with all reflected windings

approach to addressing this issue in an approximate analytical manner consists of mirroring the windings across the core surfaces, as shown in Fig. 5. Currents in the mirrored windings are responsible for creating additional $H$-fields, which add up to the fringing $H$-fields.

At any point in space the $y$ component of the $H$-field created by winding currents, assuming that current $I$ is approximately uniformly distributed across the winding, can be found from:

$$
\begin{aligned}
H_{y, \text { windings }} & =\frac{I}{2 \pi\left(l-2 t_{\text {core }}\right)} \int_{x_{1}}^{x_{2}} \frac{x d x}{x^{2}+y^{2}} \\
& =\frac{I}{4 \pi\left(l-2 t_{\text {core }}\right)} \ln \frac{x_{1}^{2}+y^{2}}{x_{2}^{2}+y^{2}}
\end{aligned}
$$

where $\sqrt{x_{1}^{2}+y^{2}}$ and $\sqrt{x_{2}^{2}+y^{2}}$ are the distances of the two ends of each winding, and $l-2 t_{\text {core }}$ is the width of the winding. As also shown in Fig. 5, reflection of the 4 winding layers across the I segment of the core is approximated by a single equivalent layer carrying $4 I$ current. This simplifying assumption is justified by the fact that the I-core mirrored layers are relatively far away from the points of interest at the top surface of the windings. The $y$ direction of the $H$-field is already taken into account in (4). Finally, the total field in the $y$-direction, $H_{\text {resultant }}$, can be obtained as the sum of the fringing fields in (1) and the $H$-fields due to the winding currents in (4).

\section{B. Optimization of orthogonal airgaps}

The conduction loss per unit length for a thin rectangular conductor is [12]:

$$
P \propto H_{y}^{2}
$$

For an orthogonally-gapped inductor, the optimization problem takes the form

$$
\begin{array}{cl}
\underset{g_{1}, g_{2}, \Delta g}{\operatorname{minimize}} & \Sigma H_{y}^{2}\left(g_{1}, g_{2}, \Delta g\right) \\
\text { subject to } & 2 g_{1}+g_{2}=2 g_{1, \text { conv }}
\end{array}
$$

where $2 g_{1, \text { conv }}$ is the gap length in the conventional structure of Fig. 2(a). The equidistant gap $\Delta g=\left(W_{c}+l\right) / 2$ is the best choice, resulting in symmetrical field cancellation on the conductor edges. To examine the effects changing gap lengths $g_{1}$ and $g_{2}, g_{1}$ is varied, and the quantity of interest $\Sigma H_{y}^{2}$ is plotted in Fig. 6 for the top winding layer, which yields the optimum $g_{1}$ and $g_{2}$. One may note how the analytical approach 


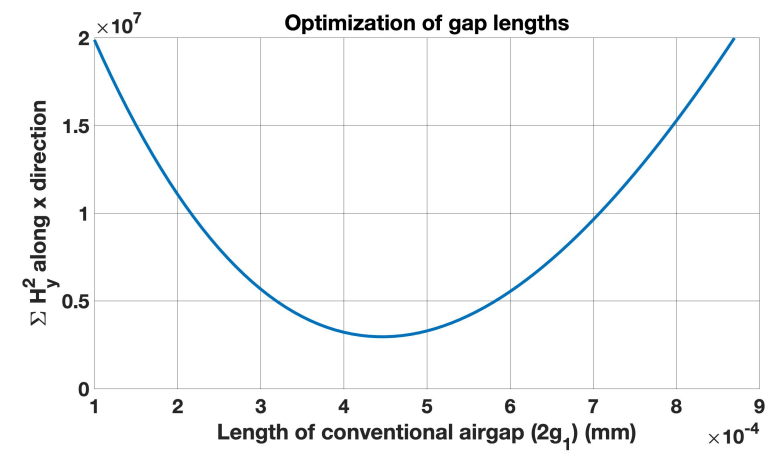

Figure 6: Optimization of gap lengths based on the perpendicular component of $H$-field.

yields a relatively simple design optimization. For the case study example, the optimum gaps are:

$$
g_{1}=0.245 \mathrm{~mm} ; g_{2}=0.38 \mathrm{~mm} ; \Delta g=10.9 \mathrm{~mm}
$$

With all the parameters selected, the perpendicular $H$ field $H_{\text {resultant }}=H_{p 1, y}-H_{p 2, y}-H_{h 1, y}+H_{y, \text { windings }}$ is plotted in Fig. 7(c) for the orhogonally-gapped inductor design. For comparison, $H_{\text {resultant }}=H_{p 1, y}-H_{p 2, y}+H_{y, \text { windings }}$ and $H_{\text {resultant }}=H_{h 1, y}+H_{y, \text { windings }}$ are also plotted for the conventional (perpendicular) airgaps and for the parallel airgap cases in Fig. 7(a) and Fig. 7(b), respectively. It can be observed that the $H$ field distribution is substantially more uniform using orthogonal gaps compared to the conventional and the parallel airgap arrangements. It should be noted that the analytical approach does not take into account the proximity effects, and the $H$ field due to the copper windings in the other window. Not considering very high frequencies, where these effects would be more pronounced, the presented model can be considered sufficiently accurate, as illustrated by the good match between the analytical results and the results of 2D finite-element simulations using Ansys Maxwell, which are overlaid in Fig. 7.

Figures 8 and 9 show 2D simulation results for the $H$ field and the current density in the three cases considered. For the conventional airgap arrangement, the numerically calculated inductance is $8.52 \mu \mathrm{H}$ and the ac resistance is $R_{a c}=44 \mathrm{~m} \Omega$. Figure 9(a) illustrates how current crowding at the two conductor edges is the main reason behind increased ac resistance in the conventional structure. The peak current density is $|J|_{\max }=14.8 \mathrm{MA} / \mathrm{m}^{2}$. For the parallel airgap case, the numerically calculated inductance is $8.32 \mu \mathrm{H}$, and the ac resistance is $R_{a c}=46 \mathrm{~m} \Omega$, which is slightly higher compared to the conventional airgaps, although the peak current density is reduced to $|J|_{\max }=5 \mathrm{MA} / \mathrm{m}^{2}$. This is because the parallel gap must be longer to obtain the same inductance. As a result, as shown in Fig. 9(b), current crowds over a wider portion in the middle of the winding layer, thus making the effective ac resistance larger. Fig. 9(c) shows how the current density is much more uniform with the orthogonally gapped core. Some current crowding still occurs at the edges, with a maximum current density of $|J|_{\max }=7 \mathrm{MA} / \mathrm{m}^{2}$, but the ac resistance drops to $R_{a c}=23 \mathrm{~m} \Omega$, which corresponds to $48 \%$

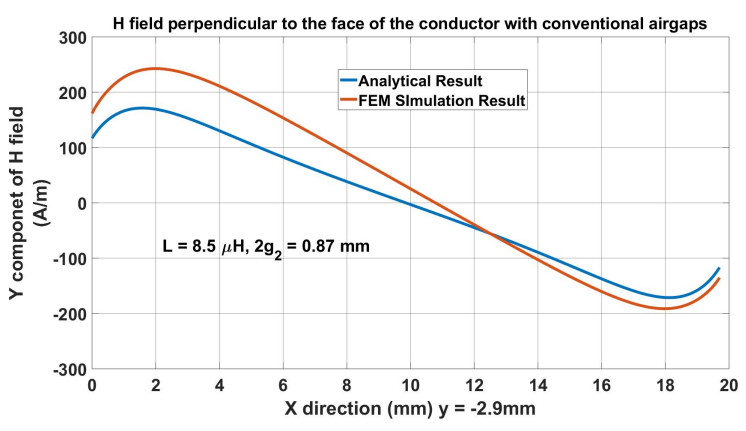

(a)

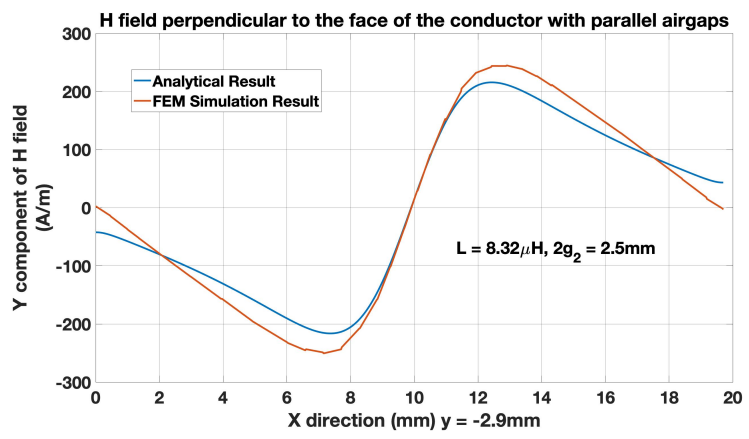

(b)

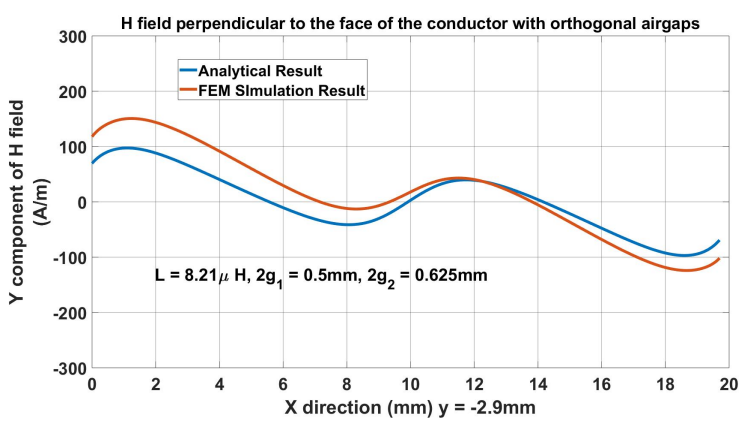

(c)

Figure 7: Comparison of analytical and 2D FEM simulation results for the distribution of the $H$ field component perpendicular to the windings in the planar inductor with (a) conventional airgaps, (b) parallel airgaps, and (c) orthogonal airgaps.

reduction in ac winding losses compared to the conventionally gapped structure, while the inductance remains approximately the same, $8.2 \mu \mathrm{H}$.

Table I summarizes 2D finite element simulation results for the three considered airgap arrangements in terms of the maximum current density and the ac resistance at two different frequencies: $100 \mathrm{kHz}$ and $250 \mathrm{kHz}$.

\section{EXPERIMENTAL VALIDATION}

An experimental prototype of the orthogonally-gapped inductor is shown in Fig. 10(b). Another inductor is made using the same PCB windings and the same core size, but with the conventional airgaps, as shown in Fig. 10(a). The inductor parameters are summarized in Section III. These inductors are used in a SiC-based $8 \mathrm{~kW}$ synchronous Buck converter operating at $250 \mathrm{kHz}$ with $50 \%$ duty ratio. To verify the predicted 


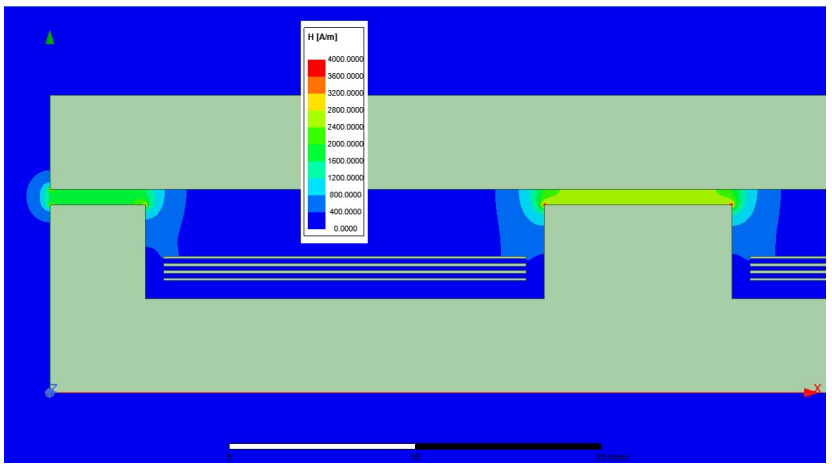

(a)

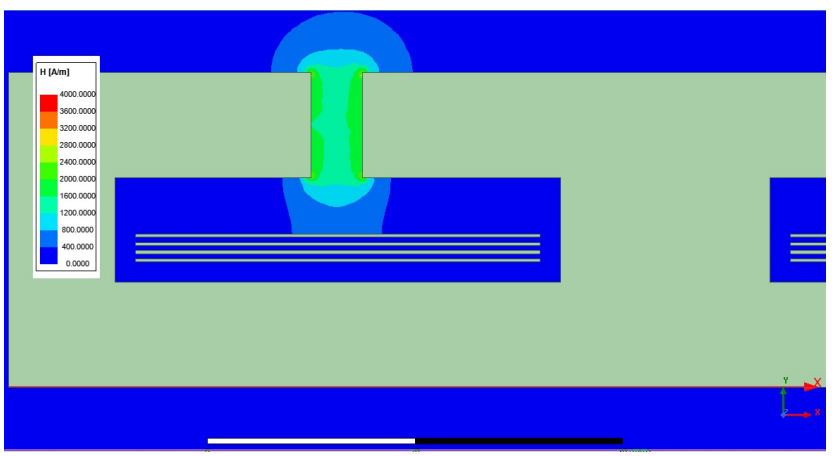

(b)

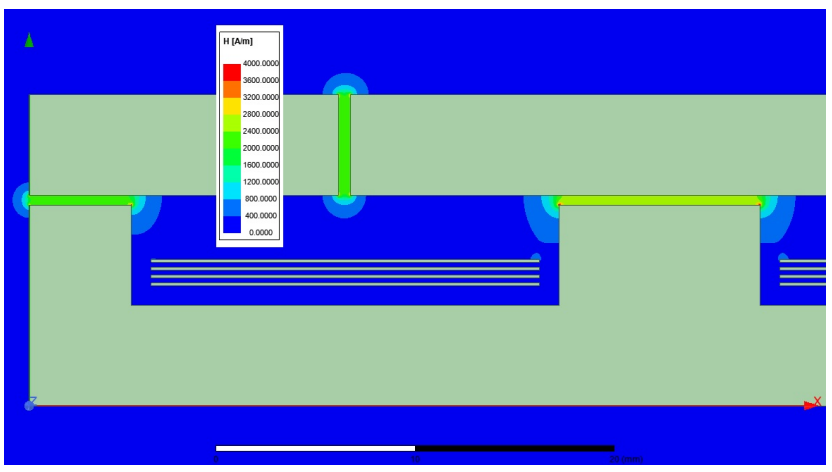

(c)

Figure 8: $H$ field distribution in the planar inductor with (a) conventional airgaps, (b) parallel airgaps, and (c) orthogonal airgaps.

Table I: Comparison of maximum current density and ac resistance in the inductor with (a) conventional airgaps, (b) parallel airgaps, and (c) orthogonal airgaps.

\begin{tabular}{l|c|c|c}
\hline Airgap arrangement & $f_{s}[\mathrm{kHz}]$ & $|J|_{\max }\left[\mathrm{MA} / \mathrm{m}^{2}\right]$ & $R_{a c}[\mathrm{~m} \Omega]$ \\
\hline \hline Conventional & 100 & 8.6 & 28.7 \\
\cline { 2 - 4 } Fig. 2(a) & 250 & 14.8 & 44.1 \\
\hline Parallel & 100 & 3.5 & 31.1 \\
\cline { 2 - 4 } Fig. 2(b) & 250 & 5.0 & 46.2 \\
\hline Orthogonal & 100 & 4.0 & 15.1 \\
\cline { 2 - 4 } Fig. 2(c) & 250 & 7.0 & 23.8 \\
\hline
\end{tabular}

loss reduction, the converter is operated unloaded, with input voltage varying from $200 \mathrm{~V}$ to $400 \mathrm{~V}$. The inductor current has a triangular shape with zero dc bias and a peak value proportional to the input voltage. The total converter loss as a function of inductor RMS current is compared in Fig. 11(a) for the conventionally-gapped inductor and for the orthogonallygapped inductor. Since all other losses are approximately the same, the experimentally-measured loss difference can

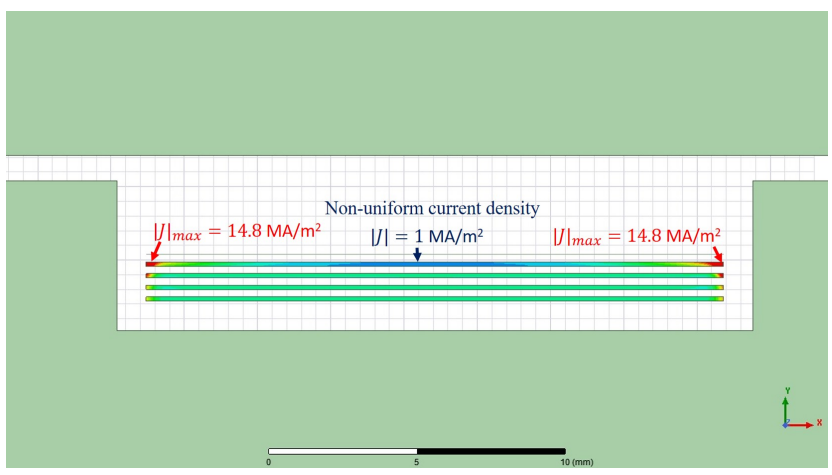

(a)

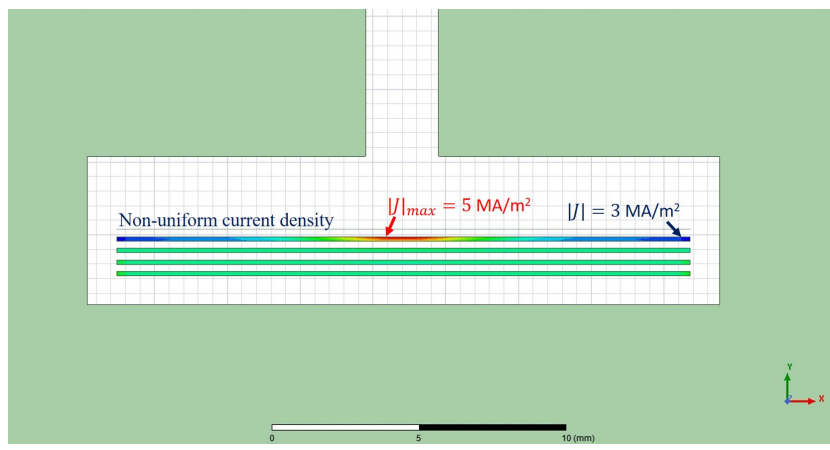

(b)

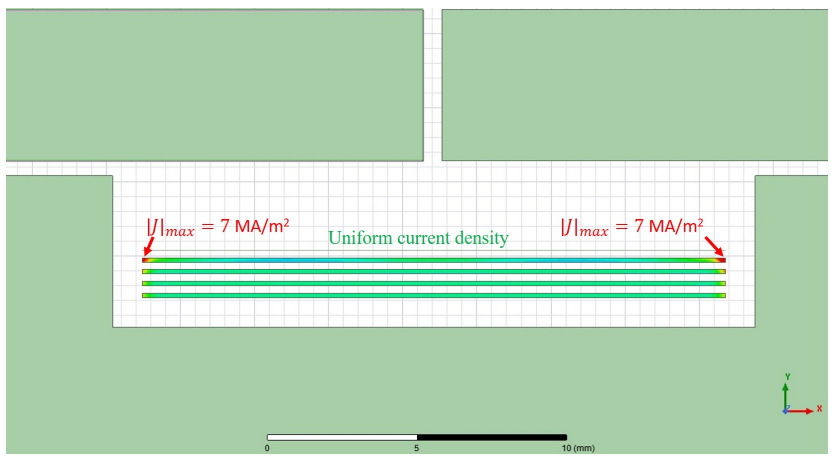

(c)

Figure 9: Current density in the planar inductor with (a) conventional airgaps, (b) parallel airgaps, and (c) orthogonal airgaps.

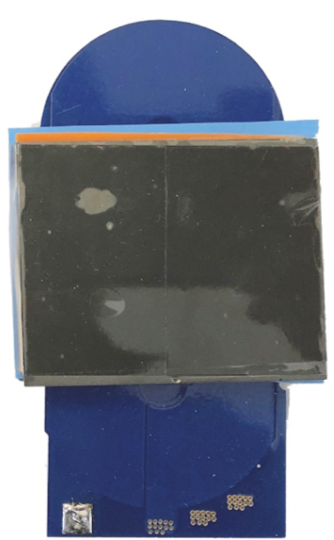

(a)

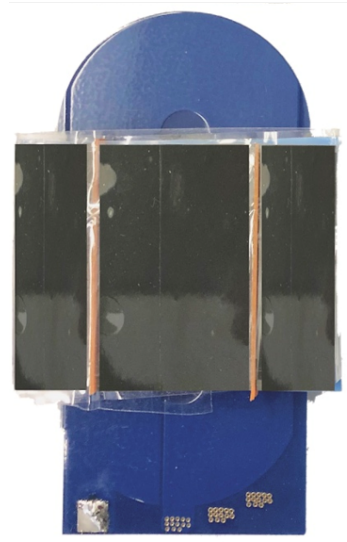

(b)
Figure 10: Planar inductor prototypes using EILP 64 core set with (a) conventional airgaps, and (b) orthogonal airgaps. 


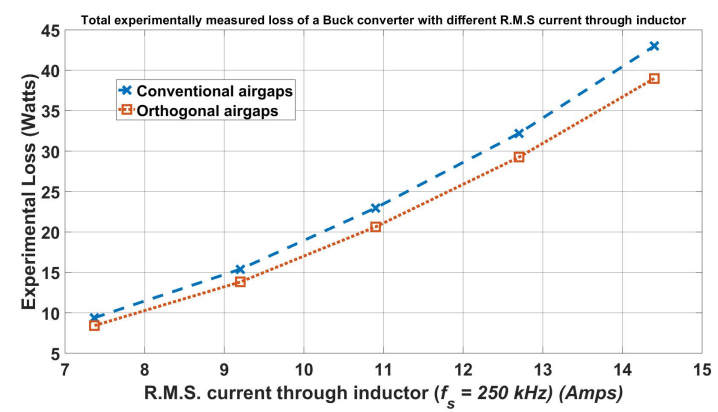

(a)

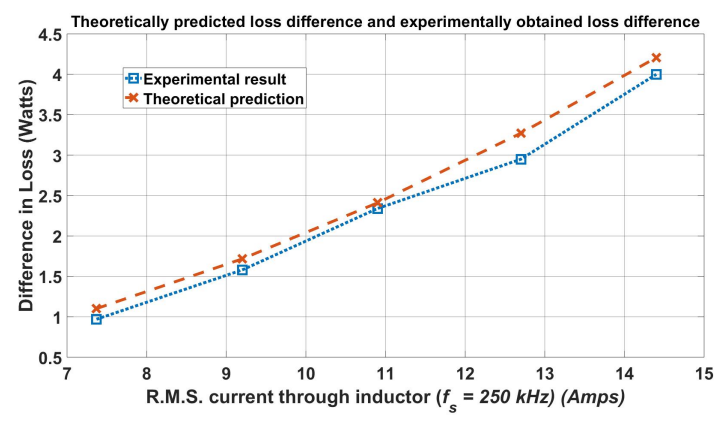

(b)

Figure 11: Comparisons of (a) total measured converter losses in the prototypes with conventional airgaps (blue), and with orthogonal airgaps (red), and (b) experimentally measured loss difference (blue), and analytically predicted loss difference (red).

be used to estimate the reduction in ac winding losses. The experimental results confirm more than $45 \%$ inductor loss reduction in the orthogonally-gapped inductor. Furthermore, the predicted difference in ac resistance calculated by $2 \mathrm{D}$ FEM simulations are listed in Table I, and the experimentally obtained differences are plotted in Fig 11(b), demonstrating a close match.

\section{CONCLUSIONS}

A simple orthogonal-gap technique is proposed to reduce effects of fringing fields in high-frequency inductors. The approach is well-suited for planar structures where it amounts to distributing airgaps between core segments perpendicular to PCB windings and segments parallel with the windings. The perpendicular airgaps are added conventionally, simply by inserting a spacer between planar core segments. A 1D analytical approach [10] is applied to derive the $H$-field distribution and optimize the gap lengths. 2D finite element simulations along with experimental results are provided to compare airgap arrangements, and to verify more than a $45 \%$ reduction in ac winding losses.

\section{REFERENCES}

[1] W. M. Chew, P. D. Evans, and W. J. Heffernan, "High frequency inductor design concepts," in PESC '91 Record 22nd Annual IEEE Power Electronics Specialists Conference, June 1991, pp. 673-678.

[2] Z. Ouyang and M. A. E. Andersen, "Overview of planar magnetic technology fundamental properties," IEEE Transactions on Power Electronics, vol. 29, no. 9, pp. 4888-4900, Sep. 2014.

[3] C. R. Sullivan, "Prospects for advances in power magnetics," in CIPS 2016; 9th International Conference on Integrated Power Electronics Systems, March 2016, pp. 1-9.

[4] B. A. Reese and C. R. Sullivan, "Litz wire in the MHz range: Modeling and improved designs," in 2017 IEEE 18th Workshop on Control and Modeling for Power Electronics (COMPEL), July 2017, pp. 1-8.

[5] J.Hu and C. R. Sullivan, "Ac resistance of planar power inductors and the quasidistributed gap technique," IEEE Transactions on Power Electronics, vol. 16, no. 4, pp. 558-567, July 2001.

[6] K. D. T. Ngo and M. H. Kuo, "Effects of air gaps on winding loss in high-frequency planar magnetics," in PESC '88 Record., 19th Annual IEEE Power Electronics Specialists Conference, April 1988, pp. 11121119 vol.2.

[7] R. A. Jensen and C. R. Sullivan, "Optimal core dimensional ratios for minimizing winding loss in high-frequency gapped-inductor windings," in Eighteenth Annual IEEE Applied Power Electronics Conference and Exposition, 2003. APEC '03., vol. 2, Feb 2003, pp. 1164-1169 vol.2.

[8] T. Ge, K. D. T. Ngo, and J. Moss, "Two-dimensional gapping to reduce light-load loss of point-of-load inductor," IEEE Transactions on Power Electronics, vol. 32, no. 1, pp. 540-550, Jan 2017.

[9] J. Schafer, D. Bortis, and J. W. Kolar, "Optimal design of highly efficient and highly compact PCB winding inductors," in 2018 IEEE 19th Workshop on Control and Modeling for Power Electronics (COMPEL), June 2018, pp. 1-8.

[10] W. A. Roshen, "Fringing field formulas and winding loss due to an air gap," IEEE Transactions on Magnetics, vol. 43, no. 8, pp. 3387-3394, Aug 2007.

[11] Jiankun Hu and C. R. Sullivan, "Optimization of shapes for round-wire high-frequency gapped-inductor windings," in Conference Record of 1998 IEEE Industry Applications Conference. Thirty-Third IAS Annual Meeting (Cat. No.98CH36242), vol. 2, Oct 1998, pp. 907-912 vol.2.

[12] E. Snelling, Soft Ferrites: Properties and Applications. Butterworth \& Co., 1988. 